\title{
An Architecture for TV Content Distributed Search and Retrieval Using the MPEG Query Format (MPQF)
}

\author{
Rubén Tous \\ UPC (Universitat Politècnica \\ de Catalunya, Spain) \\ rtous@ac.upc.edu \\ Giovanni Cordara \\ TILAB (Telecom Italia Lab)
}

\author{
Anna Carreras \\ UPC (Universitat Politècnica \\ de Catalunya, Spain) \\ Francini Gianluca \\ TILAB (Telecom Italia Lab)
}

\author{
Jaime Delgado \\ UPC (Universitat Politècnica \\ de Catalunya, Spain) \\ Enric Peig \\ UPF (Universitat Pompeu \\ Fabra, Spain)
}

\begin{abstract}
Traditional broadcasting of TV contents begins to coexist with new models of user aware content delivery. The definition of interoperable interfaces for precise content search and retrieval between the different involved parties is a requirement for the deployment of the new audiovisual distribution services. This paper presents the design of an architecture based on the MPEG Query Format (MPQF) for providing the necessary interoperability to deploy distributed audiovisual content search and retrieval networks between content producers, distributors, aggregators and consumer devices. A service-oriented architecture based on Web Services technology is defined. This paper also presents how the architecture can be applied to a real scenario, the XAC (Xarxa IP Audiovisual de Catalunya, Audiovisual IP Network of Catalonia). As far as we know, this is the first paper to apply MPQF to TV Content Distributed Search and Retrieval.
\end{abstract}

\section{Categories and Subject Descriptors}

H.3.3 [Information Storage and Retrieval]: Information search and Retrieval; D.2.11 [Software Engineering]: Software-domain-specific architectures

\section{Keywords}

information retrieval, multimedia retrieval, distributed information retrieval, MPQF, MPEG Query Format, TV

\section{INTRODUCTION}

Nowadays, the consumption paradigm of TV programs is changing due to the availability of broadband IP access, return channels and rich media applications providing some ways of interaction. The traditional "push" mode user experience is merging with the more interactive one typical of $\mathrm{PCs}$, and the fruition of video contents in both these worlds

Permission to make digital or hard copies of all or part of this work for personal or classroom use is granted without fee provided that copies are not made or distributed for profit or commercial advantage and that copies bear this notice and the full citation on the first page. To copy otherwise, to republish, to post on servers or to redistribute to lists, requires prior specific permission and/or a fee.

Ambi-sys '08 February 11-14, 2008, Quebec, Canada

Copyright 2008 ACM ...\$5.00. is changing towards a "pull" model, where users can be more active, searching videos to enjoy among a huge amount of contents.

DVB-T with return channel or IPTV platforms may provide services able to introduce new functionalities: unicast distribution to the audience based on single requests; TV quality through the IP network; higher number of TV channels and the availability of any kind of on-demand content, accessing both web sites and servers over the Internet or a large repository of TV-formatted contents.

The convergent access to servers over the internet and TV content repository gives the opportunity to make a large amount of multimedia contents previously accessible only to a little number of people, available to the whole TV audience. These new kinds of contents can be divided into two different categories:

- Niche contents: as reported by Anderson [2], studies on TV audience show that people make a massive consumption of few television channels, the so called "Long Tail" concept, fig. 1. This means that $80 \%$ of the audience watches about $20 \%$ of the available contents, which may not necessarily be the best, the most interesting or the ones of highest artistic value. TV service providers, therefore, can leverage the new opportunity raised by the spread of niche contents offering to their users refined and innovative videos to enjoy;

- User generated contents: the affordability of consumer electronic devices such as MP3 recordable players, digital cameras, DV camcorders, and smart phones allows people to produce and share a huge number of contents.

The rising tide of available contents creates the need of new tools for guiding users to find what is of their interest. Meanwhile, exploring the contents to search for the one to enjoy is becoming part of the entertainment itself: besides accessing to an heterogenous amont of contents, viewers might have the opportunity to navigate among contents through dynamic and non-linear solutions that can improve their user experience. This results in the need of effective search and retrieval platforms that have to fulfill the following requirements:

- Interoperability: service providers can rely on platforms drawing contents by many different ownership 


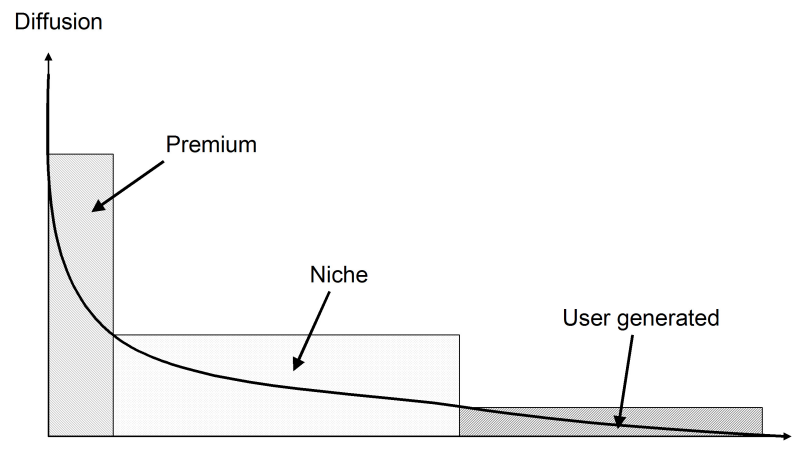

Figure 1: The "long tail" concept

domains; therefore, a standard way to access and search among contents, would help in the design of distributed architectures;

- Flexibility: search and retrieval engines shall offer to the users different ways to interact, express different kind of queries and go beyond the traditional and well known textual query (with different search criteria, as query by example, query by segment, simple browsing $\check{E})$.

An effort to address these two requirements is provided by the emerging MPEG Query Format (MPQF) standard [19, $1,8]$, which provides a unified interface to multimedia databases.

The paper describes a service-oriented architecture based on Web Services technology allowing users to express their search criteria and receiving the results in the desired format accommodating the search and retrieval of contents in a format compliant to the emerging standard. The paper is organized in the following way: a description of the related work is reported in Section 2. Section 3 contains a description of the architectural requirements. Then the referred architecture is described (Section 4). Finally, the application of the proposed architecture in a real scenario is described in section 5 .

\section{RELATED WORK}

Research around the definition of a unified multimedia query language has been refuelled by the initiative of standardization of an MPEG Query Format (MPEG) taking place within the MPEG context (ISO/IEC JTC1/SC29/ WG11). The process started in July 2006 with the release of a "Call for Proposals on MPEG-7 Query Format" (ISO/ IEC JTC1/SC29/WG11 N8220). A proposal [16] containing parts of the research work presented in this paper was submitted in January 2007 as a response to the Call.

With respect to previous works, MPQF can be applied to MPEG-7 as $[6,15,10,9,7]$ but also to other metadata formats. MPQF outperforms XQuery-based approaches like [9, $15]$ because, while offering the same level of expressiveness, it offers multiple content-based search functionalities (QBE, query-by-example) and other Information Retrieval-like features (e.g. paging, relevance feedback, personalization). It differs from high level approaches like $[7,10,6]$ because it keeps working over the data model defined by MPEG-7 or any other existing metadata format, and does not attempt to define a higher-level data model.
With respect to service-oriented architectures for multimedia retrieval, existing research is very limited $([21,14])$, and there are no works based on Web Services technology.

As far as we know, this is the first paper to apply MPQF to TV Content Distributed Search and Retrieval.

\section{ARCHITECTURE REQUIREMENTS}

A service-oriented Architecture for TV Content Distributed Search and Retrieval can benefit from the adoption of a standard query language like the MPEG Query Format: the emerging standard well suits the requirements of the referred application scenario, since it provides a unified query format, supporting different search criteria and accommodating user preferences and desired output formats.

An architecture adopting this standard can provide a unified and standard way to access and interrogate distributed multimedia databases (MMDBs), while maintaining the flexibility in the different search criteria allowed by the query format. Some of the requirements of this platform, therefore, simply inherit from those fulfilled by the MPQF standard, shifting the problem on a higher level and moving from the query format point of view to the architectural point of view. In deeper details, the described platform shall allow the following operations:

- Supporting different search criteria and providing user interfaces to allow their execution:

- Query by description;

- Query by example;

- Browsing (Empty Search);

- Compositional Search;

- Segment-based Search

- The search and retrieval platform shall allow users to compose a query condition by combining multiple constraints using simple operands, operation types and expression types;

- User profiles shall be taken into considerations, influencing the search results according to their preferences;

- Output query format shall fit the chosen parameters, even adapting to display features of the rendering device. The output results shall be paged, sorted or grouped;

- The search engine shall provide advanced functionalities to help users in their Information Retrieval (IR) operations, like relevance feedback and searching within previous results.

Finally, the real implementation of a distributed platform that rely on different distributed databases and aims at maintaining the access procedures transparent to the user presents an extra requirement: binding the query format to different technologies to grant flexibility and allowing the easy integration with different domains. Each domain can, in fact, be accessed in different ways, still maintaining compatibility of the exchanged data with the MPQF standard.

These architectural aspects have been taken into consideration in the study of the proposed architecture, described in the next section. 


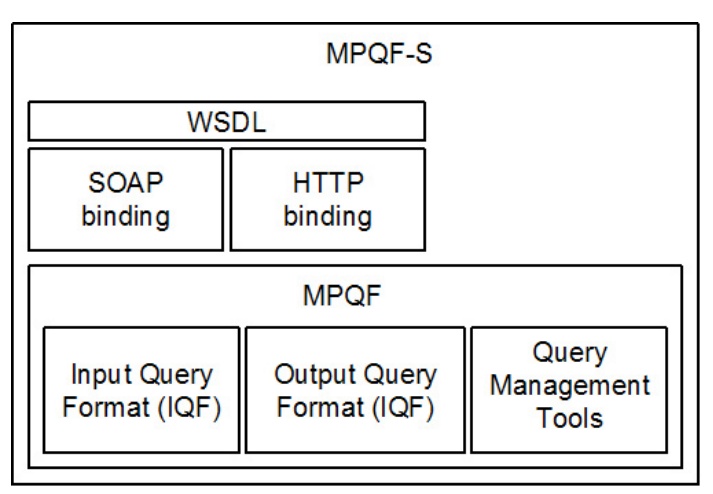

Figure 2: Architecture overview

\section{ARCHITECTURE DESCRIPTION}

The definition of an uniform query language like MPQF is just one of the steps to achieve a real interoperability between multimedia repositories. It defines a unified way to query multimedia databases in the most flexible way, without any constraints or assumptions about the target scenarios. However, because not all repositories need to be interested or need to be capable of exposing all possible query functionalities, a service interface layer is needed. This higher level layer will allow selective service level adoption, and will also simplify the task of aggregators and distributed search engines.

If we want users experiencing aggregated services from various MPQF compliant databases we need to define a unified and low-barrier service interface over our query language as it exists in the Digital Libraries discipline. Experiences like the Protocol for Metadata Harvesting (PMH) of the Open Archives Initiative (OAI) [11], or the SRW/SRU languages of the Z39.50 Maintenance Agency, show that simple and low-barrier interfaces are best suited for a distributed search or aggregation scenario. So, we need to define a low-barrier and scalable interface that allows new and old repositories to expose their contents by selecting a subset of query functionalities, which could range from simple metadata aggregation to complex query-by-example searches. We suggest (see figure 2):

1. The use of the MPEG Query Format (MPQF) as a uniform way to achieve interoperability between distributed audiovisual repositories.

2. The definition of an extensible Web Services-based (SOAP) [13] interface for querying and managing audiovisual contents described with MPEG-7 or any other metadata format. Each exposed functionality will be represented by an operation (in Web Services terminology). Operations will act as views over the MPQF language, limiting the expressivity of the MPQF queries for a specific use case.

3. An alternative and simple HTTP (REST-like [12]) interface (being the request a simple URI and the response an XML file) when possible and just for operations involving searches compliant with the SOAP 1.2 web-method recommendation (idempotent, free of side effects, etc.).

\subsection{MPEG Query Format (MPQF)}

\subsubsection{Unified multimedia query languages and inter- faces}

Within the process of searching and retrieving audiovisual materials, user information needs can be expressed in many different ways. On the one hand, search preferences can be expressed in terms of precise conditions, as those in a relational algebra expression, clearly determining which objects of a collection to select. For a data retrieval (DR) system, like a database, a single erroneous object among a thousands retrieved means a total failure. So, data retrieval deals with well defined data models, expressive query languages and performance issues. In the context of multimedia search and retrieval, DR refers to queries expressed in terms of metadata and also in terms of low-level features.

On the other hand, there are user information needs which cannot be easily formalized. Information Retrieval (IR) aims to retrieve information which might be relevant to the user, given a query written from the user point-of-view. In the context of multimedia search and retrieval, IR refers to text keywords and query-by-example (QBE) for instance.

Today, digital contents are often composite of the contents themselves and also machine understandable metadata. Querying such complex objects can imply the combination of data retrieval-like conditions referred to a well defined data model and also information retrieval-like conditions.

Many modern MMDBs and many providers of multimedia search and retrieval services already offer advanced indexing and retrieval techniques for multimedia contents. However, their databases and service interfaces are proprietary and therefore the solutions differ and do not interoperate. The definition of unified languages and interfaces to accept and respond to requests for multimedia contents searches would facilitate multimedia repositories interoperability. Interoperability would ease the access to repositories by users and applications, and would allow the deployment of distributed search and aggregation services.

\subsubsection{Metadata standards and the MPEG Query For- mat (MPQF) for distributed multimedia search and retrieval}

Existing metadata standards as the already completed ISO/IEC IS 15938, formally called the Multimedia Content Description Interface (better known as MPEG-7), provide rich models for multimedia content description. The usage of such standards should guarantee interoperability at DR level. Generally, metadata descriptions link to standard schemas and should be interoperable with respect to XML query languages like W3C's XQuery [18].

However, as pointed above, data retrieval is not the only feature of a modern multimedia database. Some contentbased search and retrieval constraints (e.g. query-by-example or confidence management) must be also combined with the use of metadata descriptions. Furthermore, some multimedia metadata descriptors, as those from MPEG-7, encapsulate low-level features (e.g. a colour histogram) which contain data, but these data are only useful if they can be queried and processed with specific content-based functionalities (e.g. various similarity functions). In this sense, XQuery does not provide specific normative functionalities for query-by-example or in general for content similarity 


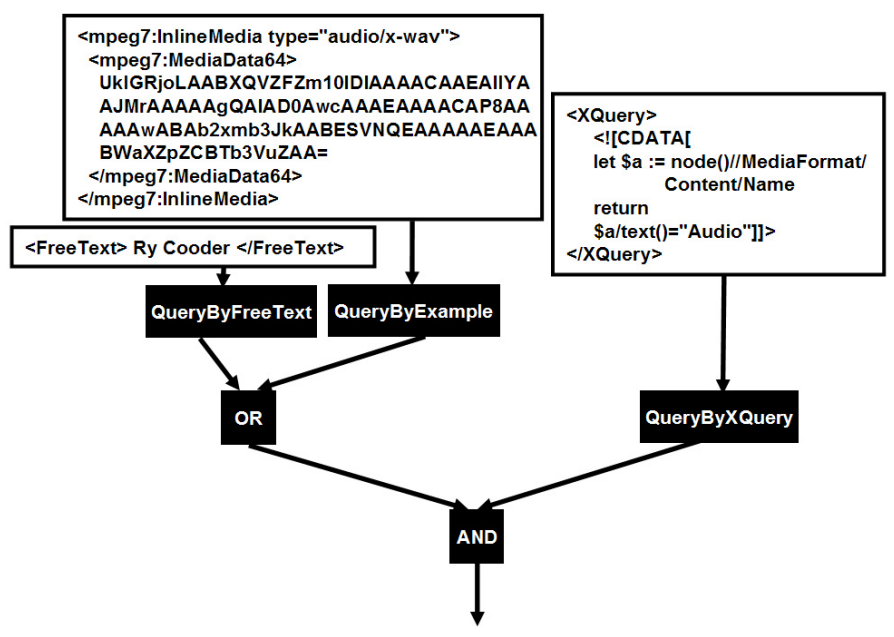

Figure 3: Example combination of query types

measurement. A language which combines the expressive power of XQuery with content-based functionalities (e.g. an extension of XQuery) could be a good candidate for an unified query language for multimedia databases. MPEG is now finishing the standardization of a language with these features, the MPEG Query Format (MPQF). The adoption of MPQF could raise interesting benefits from the multimedia applications development point of view, in a similar way in which SQL did for applications based on relational data platform independence, reusability, maintainability, etc.

\subsubsection{MPQF overview}

MPQF is an XML-based language which defines the format of queries and replies to be interchanged between clients and servers in a multimedia information search and retrieval environment. MPQF can be used in standalone MMDBs, but it has been specially designed for scenarios in which several MMDBs and content aggregators interact. MPQF does not make any assumptions about the metadata formats used by the target MMDBs, which can be MPEG-7 but also any other format (Dublin Core for example).

MPQF allows combining Information Retrieval (IR) criteria with Data Retrieval (DR) criteria. While IR deals with user information needs which cannot be easily formalized (e.g. keywords, query-by-example, etc.), DR deals with well defined data models (e.g. XML) and clearly defined conditions (e.g. range of values of a metadata field). For the DR criteria, MPQF offers its own XML query algebra, but also offers the possibility to embed XQuery [18] expressions.

A valid MPQF document (according to the MPQF XML schema) includes always the MpegQuery element as the root element. Below the root element, an MPQF document includes the Input element or the Output element, depending if the document is a client query or a server reply (there's only one schema and one root element). The part of the language describing the contents of the Input element is usually named the Input Query Format (IQF). The part of the language describing the Output element is usually named the Output Query Format (OQF). IQF and OQF are just used to facilitate understanding, but do not have representation in the schema.

\subsubsection{Input Query Format (IQF)}

The IQF mainly allows specifying the search condition tree and also the structure and desired contents of the server output. The condition tree is the main component of MPQF, and can be built combining operands, operation types, expression types and query types.

- Operands (groups to be used as input for the operation types and expression types): ArithmeticOperands, BooleanOperands, StringOperands, DateTimeOperands and DurationOperands.

- Operation types (allow to build expressions which result in a boolean value): Boolean operations (AND, OR, NOT, XOR) and comparison operations (GreaterThan, GreaterThanEqual, LessThanEqual, LessThan, Equal, NotEqual, Contains).

- Expression types (allow to build expressions which result in a non-boolean value): arithmetic expressions (Add, Subtract, Multiply, Divide, Modulus, Abs, Ceiling, Floor, Round), string expressions (UpperCase, LowerCase).

- Query types (QueryByExample, QueryByXQuery, QueryByRelevanceFeedback and QueryByFreeText)

The graphical example in Figure 3 shows an MPQF condition tree. The Boolean operations can be used to combine conditions expressed using different query types like the QueryByFreeText, the QueryByExample or the QueryByXQuery. The presented query specifies the need to find audio contents similar to a given example WAV file (embedded within the query in base64 encoding) and related to the free-text "Ry Cooder". Example in Code 1 shows an example of the QueryByExample QueryType. The request includes an example audio file (in WAV format), which is directly embedded within a Resource element using base64 encoding. Instead of inlining the example within the Condition element (which is also possible), it is defined within the QFDeclaration section. This would allow, for example, reusing the same data for other conditions just by referring to the same id. It is up to the server to decide which similarity measure and search algorithm to apply. Within the IQF, but with respect to the output format specification, MPQF relies on XPath to offer flexibility when defining the structure of the XML result set. On the other hand, the OQF specifies the valid format of the server responses, which have a fixed part defined by MPQF and also a placeholder where MMDBs can return metadata in any XML format. Of course this is a basic overview of MPQF, which offers many other features like grouping, sorting, user preferences, relevance feedback, asynchronous mode, searching within previous results, and several query management tools like service capabilities description and selection.

\subsubsection{Output Query Format (OQF)}

The OQF specifies which are the valid outputs from the server to the client. The responses from an MPQF compliant server will always include a sequence of records (under the ResultItem tag), and within each record the client can find several MPQF standard fields like the URI of the related content or a text field, but also a complete or partial metadata description in an external format like MPEG-7. Example in Code 2 contains a complete example response. 


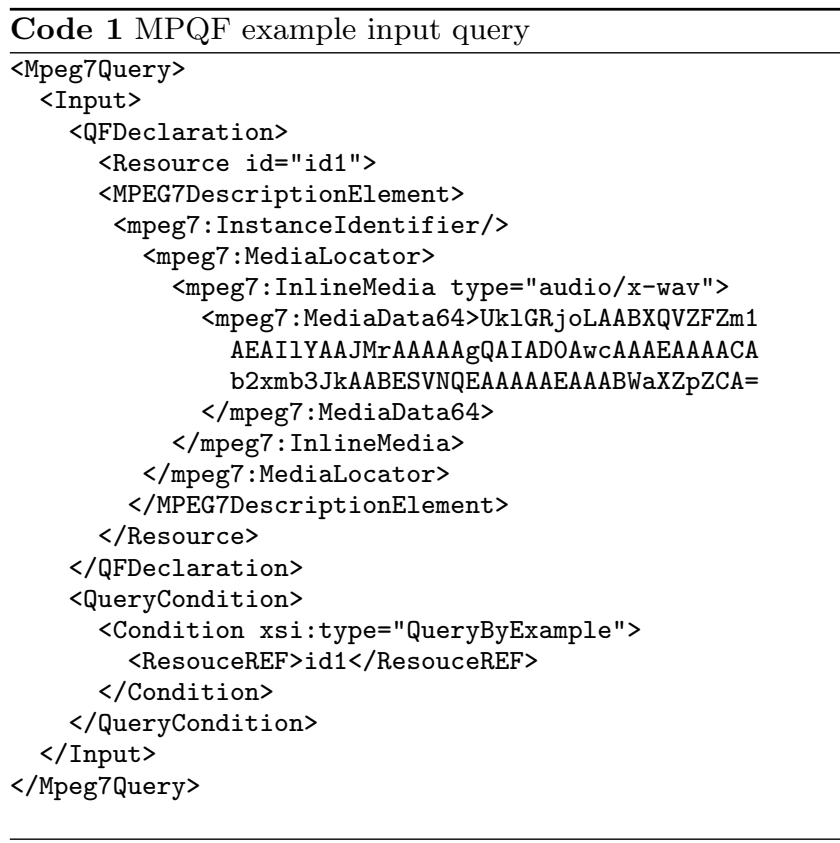

\subsubsection{MPQF standardization progress}

After the 82nd MPEG meeting in Shenzhen, China (2007$10-22 / 26)$

The standardization work is progressing and recently a Final Committee Draft (FCD), containing important improvements, has been approved (during the 82nd MPEG meeting in Shenzhen, China, October 2007). The improvements are related to:

- Clarification and potentially extension of the Base Data Model.

- Definition of new Query Types.

- Syntactic definition of the Join operator.

At the current stage, no more technical changes are expected, and only minor editorial corrections can be done. It is expected that MPQF will become an ISO standard for mid 2008 .

\subsection{MPQF service interface (MPQF-S)}

We define the MPQF service interface (MPQF-S) as a unified service interface over MPQF to accept and respond to user requests in a distributed multimedia search and retrieval scenario (see figure 5). As pointed above, audiovisual search and retrieval systems offer different levels of functionality which are suitable for being standardized (see figure 4).

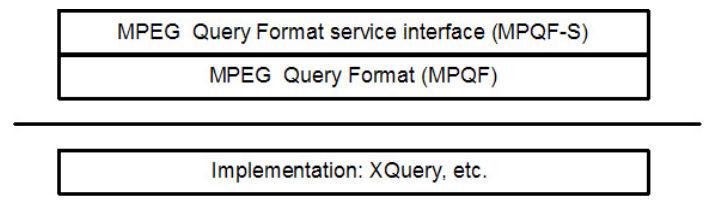

Figure 4: Proposed MPQF querying layers
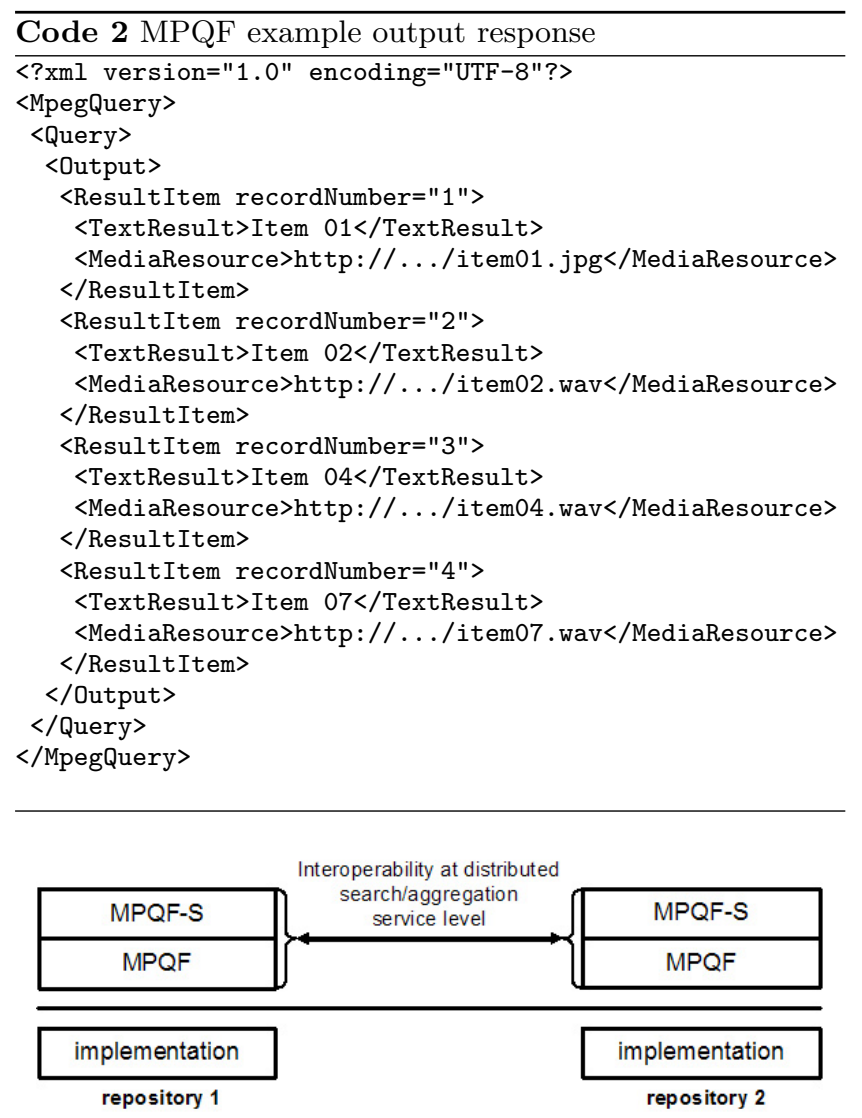

Figure 5: Example scenario

\subsection{SOAP and HTTP bindings}

MPQF-S is a service defined through two different bindings, the W3C's SOAP protocol (Simple Object Access Protocol) [13] which is currently at version 1.2 , and a raw usage of the HTTP protocol and XML.

All MPQF-S operations are defined as Web Services operations and described in WSDL 2.0. All operations offer a SOAP 1.2 binding with both, request and response, expressed in XML. Additionally, some operations offer also an HTTP binding which will allow embedding the request inside an URI. This URI-based invocation adheres to the SOAP 1.2 - Part 2 section 4.1 recommendations and follows the Representational State Transfer (REST) architectural style [12]. These two possibilities have been chosen following the model of the SRW/SRU languages of the Z39.50 Maintenance Agency, which combine some features of the ISO 8601:1988 (Z39.50) [20].

\subsubsection{Queries within URIS (MPQF-URI)}

We have included a special serialization of a limited version of MPQF for operations that can be invoked through the HTTP binding. The MPQF-URI syntax is inspired on the syntax of CQL [3], the Common Query Language, in combination with some datatypes and functions defined for XQuery. CQL is a formal language for representing queries to Information Retrieval systems developed by the Z39.50 (ISO 23950) Maintenance Agency as part of its ZING initiative ("Z39.50-International: Next Generation"). CQL combines the simplicity of intuitive languages like Google search- 
ing with the expressive power of the more complex languages like SQL, W3C's XQuery or the Z39.50 Type-1 query. An example instance of MPQF-URI could be:

mediaType=VideoType and (creationDate=1899-12-30

or creatorFamilyName=Smith)

When sending a request through HTTP GET, with the L7URI instance embedded within the query part of an URI, some characters must be percent-encoded as defined in RFC 3986. The previous example will be converted to:

http://www. someprovider.com/mp7qf/REST/

getMultimediaByTextualDescription?

where=mediaType\%3DVideoType\%20and (creationDate\%3D1899-12-30

$\% 20$ or\%20creatorFamilyName\%3DSmith

\subsubsection{List of operations}

We have partitioned the MPQF functionality in a set of independent operations. MPQF querying defines a multidimensional space of functionalities, which have 1) different levels of deployment difficulty (e.g. keywords-based querying can be easier to deploy than a query-by-example functionality), 2) different contexts and domains (e.g. audio content providers and video content providers) and 3) transversal aspects (e.g. user authentication or digital rights management). Appendix A enumerates the list of defined operations.

\section{A REAL SCENARIO. XAC, AUDIOVISUAL IP NETWORK OF CATALONIA}

The architecture presented in this paper is currently being instantiated for a real scenario, the XAC project [4] (Xarxa IP Audiovisual de Catalunya, Audiovisual IP Network of Catalonia). XAC is a regional project, funded by the Catalan government, and led by researchers of the DMAG (Distributed Multimedia Applications Group) [5]. The aim of the project is to create a new marketplace for television content providers, defining mechanisms for searching, cataloguing and selling television programs in a secure way. Currently local TV channels are the main users of the system, but other multimedia content producers (not only content for TV distribution but also, for example, for learning purposes) are also participating and being taken into account.

One of the most important challenges of XAC is the interoperability of metadata. The first step taken was the analysis of the way of work of more than twenty local TV's. The result of this analysis was a set of common metadata used by the participants of the project in order to classify their contents. Not only the common fields of the metadata were defined, but also the semantics for these terms, in order to allow content search and interchange in a near future. Our objective was then to decide a common schema based on an international standard perfectly adapted to the needs of the local channels. It should be simple but complete. This common schema is called the XAC Metadata Set (XAC-MS).

XAC-MS separates between public and private fields because some fields are only relevant to the local TV that recorded the program, so they prefer not to make it public to the rest of TV producers that could be interested on the program. Table 1 shows the fields inside the XAC-MS public part. In order to assure the semantic interoperability and to avoid misspelling, we proposed to use a controlled vocabulary for the elements genre, format, language, subtitles,
Table 1: XAC-MS public fields equivalence with MPEG-7

\begin{tabular}{|l|l|}
\hline XAC metadata set & MPEG-7 \\
\hline \hline Title & Title \\
\hline Genre & Genre \\
\hline Format & AVAtributes \\
\hline Duration & AVAttributes \\
\hline Language & Language \\
\hline Subtitles & Caption Language \\
\hline Edited & Related Material \\
\hline Ticker & Related Material \\
\hline Shooting date & ProductionDate \\
\hline Editing date & CreationCoordinates Date \\
\hline Broadcasting date & Release Date \\
\hline Keywords & Keywords \\
\hline Synopsis & Synopsis \\
\hline Target audience & Intended Audience \\
\hline Awards & Awards \\
\hline Observations & Related Material \\
\hline Credits & Related Material \\
\hline Credit list & Structured Annotation \\
\hline
\end{tabular}

edited, ticker, intended audience and credits. We created the initial lists of possible terms from the information recorded by all the providers, alingning similar terms to a unique one, following the Classification Schemas (CS) used by MPEG-7. The XAC project is specially suited as a test environment for MPQF-S. XAC guarantees that the target TV contents will be annotated using the XAC Metadata Set (XAC-MS). This allows to test all the range of possibilities of MPQF-S, from IR-like searches, to DR-like queries, as those dealing with XQuery expressions. Now, we are working to deploy instances of MPQF-S over XAC nodes. The process needs to be taken in parallel to the MPQF implementation, in which we are also involved.

\section{CONCLUSIONS AND FUTURE WORK}

The work presented in this paper shows how an architecture based on the MPEG Query Format can be applied to deploy distributed TV content search and retrieval services between content producers, distributors, aggregators and consumer devices.

We describe a service-oriented architecture based on Web Services technology which acts as a set of views over the query language layer. The defined interface offers two different bindings: An extensible Web Services-based (SOAP) [13] binding and an alternative and simple HTTP (RESTlike [12]) interface.

Currently we have a partial implementation of a reference query processor and we are working to release a complete reference implementation for both, a query processor and the service interface (with the SOAP and HTTP bindings). In parallel the work is being discussed under the standardization process of an MPEG Query Format (MPQF), taking place within the MPEG context (ISO/IEC JTC1/ SC29/WG11). Parts of this work were submitted as a response to the MPEG call for proposals [17] and parts of the submission were selected to be part of the standard. It is expected that the MPEG Query Format (MPQF) will become an ISO standard after the 85th MPEG meeting in Hannover, Germany, July 2008.

We describe also how the architecture can be applied to a real scenario, the XAC (Xarxa IP Audiovisual de Catalunya, Audiovisual IP Network of Catalonia), proving the value of this novel approach which could open a new research an- 
gle for distributed multimedia information retrieval. As far as we know, this is the first paper to apply MPQF to TV content search.

\section{ACKNOWLEDGMENTS}

This work has been partly supported by the Spanish government (DRM-MM project, TSI 2005-05277) and the European Network of Excellence VISNET-II (IST-2005-2.41.6) under the European Commission IST 6th Framework Program.

\section{ADDITIONAL AUTHORS}

Additional authors: Frederic Dufaux (EPFL (Ecole Polytechnique Fédérale de Lausanne)) and Grzegorz Galinski (IPW (Warsaw University of Technology, Poland)).

\section{REFERENCES}

[1] K. Adistambha and al. The MPEG-7 Query Format: A New Standard in Progress for Multimedia Query by Content. In 7th International Symposium on Communications and Information Technologies (ISCIT 2007), Sydney, Australia, October 16-19, 200\%. IEEE Computer Society Press, 2007.

[2] C. Anderson. The Long Tail. Forget squeezing millions from a few megahits at the top of the charts. The future of entertainment is in the millions of niche markets at the shallow end of bitstream. Wired Magazine, 12(10):86-103, 2004.

[3] Common query language. See http://www.loc.gov/ z3950/agency/zing/cql/cql-syntax.html.

[4] J. Delgado, S. Llorente, E. Peig, and A. Carreras. A multimedia content interchange framework for TV producers. In 2nd AXMEDIS Conference. IEEE Computer Society Press p. 206-213., 2007.

[5] Distributed multimedia applications group (dmag). See http://research.ac.upc.edu/dmag; http://dmag.upf.edu.

[6] N. Fatemi and al. An xquery adaptation for mpeg-7 documents retrieval. See http://www.idealliance.org/ papers/dx xml03/papers/05-03-01/05-03-01.html.

[7] A. Graves and M. Lalmas. Video retrieval using an mpeg-7 based inference network. In ACM SIGIR'02, August 2002.

[8] M. Gruhne, R. Tous, M. D. Mario, J. Delgado, and H. Kosch. MP7QF: An MPEG-7 Query Format. In 3rd AXMEDIS Conference, Barcelona, November 2007. IEEE Computer Society Press, 2007.

[9] J. Kang and al. An xquery engine for digital library systems. In 3rd ACM/IEEE-CS Joint Conference on Digital Libraries, Houston, Texas, May 2003.

[10] P. Liu and L. Suhsu. Queries of digital content descriptions in mpeg-7 and mpeg $21 \mathrm{xml}$ documents. In XML Europe 2002, Barcelona, Spain, May 2002.

[11] The open archives initiative. See http://www.openarchives.org/.

[12] Representational State Transfer (REST), Roy Thomas Fielding, Author. 2000.

[13] Soap version 1.2 part 1: Messaging framework. w3c recommendation 24 june 2003. See http://www.w3.org/TR/SOAP/.
[14] U. Srivastava and al. Query Optimization over Web Services. In 32nd VLDB Conference - Volume 32, pages 355-366, Seoul, Korea, 2006.

[15] D. Tjondronegoro and Y. Chen. Content-based indexing and retrieval using mpeg-7 and xquery in video data management systems. World Wide Web: Internet and Web Information Systems, pages 207-227, 2002.

[16] R. Tous and J. Delgado. L7, an MPEG-7 Query Framework. In 3rd AXMEDIS Conference, Barcelona, November 2007. IEEE Computer Society Press, 2007.

[17] R. Tous, J. Delgado, E. Peig, E. Rodríguez,

A. Carreras, G. Cordara, F. Gianluca, F. Dufaux, and G. Galinski. Mpeg-7 search and retrieval web service: A response to the mpeg-7 query format call for proposals., January 2007. ISO/IEC JTC1/SC29/WG11 MPEG2007/M14117.

[18] Xquery 1.0: An xml query language. w3c proposed recommendation 21 november 2006. See http://www.w3.org/TR/xquery/.

[19] K. Yoon, M. Doeller, M. Gruhne, R. Tous, M. Sano, M. Choi, T.-B. Lim, J. J. Lee, and H.-C. Seo. Information technology - multimedia content description interface - part 12: Query format. iso/iec 15938-12, 2004

[20] Z39.50. See http://www.ietf.org/rfc/rfc2616.txt.

[21] J. Zhang and J.-Y. Chung. An open framework supporting multimedia web services. Multimedia Tools and Applications, 30(2):149 - 164, 2006.

\section{APPENDIX}

\section{A. SERVICE INTERFACE OPERATIONS LIST}

- Generic low-barrier operations (all can be instantiated by MPQF)

- abstractRetrieval: Abstract operation (cannot be directly instantiated) from which all the retrieval operations inherit.

- getMultimedia: Low-barrier harvesting operation (OAI-like) without filtering conditions (REST enabled).

- getMultimediaBytTextualDescription: Generic fieldbased retrieval operation (REST enabled).

- getMultimediaByKeywords: Low-barrier keywordsbased operation (REST enabled).

- Image specific operations (all can be instantiated by MPQF)

- getImageByExampleDescription: Image QBE based on example XML metadata.

- getImageByExampleImage: Image QBE based on example still image.

- getImageByExampleAudio: Image QBE based on example audio file.

- getImageByExampleSegment: Image QBE based on example still image file plus segment locator.

- Video specific operations (all can be instantiated by MPQF)

- getVideoByExampleDescription: Video QBE based on example XML metadata. 
- getVideoByExampleKeyframe: Video QBE based on example still image.

- getVideoByExampleVideo: Video QBE based on example video file.

- getVideoByExampleAudio: Video QBE based on example audio file.

- getVideoByExampleSegment: Video QBE based on example video file plus segment locator.

- Audio specific operations (all can be instantiated by MPQF)

- getAudioByExampleDescription: Audio QBE based on example XML metadata.

- getAudioByExampleAudio: Audio QBE based on example audio file.

- getAudioByExampleImage: Audio QBE based on example still image.

- getAudioByExampleSegment: Audio QBE based on example audio file plus segment locator.

- Semantic specific operations (cannot be instantiated by MPQF)

- getMultimediaBySemantics: Semantic-based retrieval operation, based on some semantic query language like SPARQL.

- Update specific operations (cannot be instantiated by MPQF)

- abstractModify: Abstract operation (cannot be directly instantiated) from which all the update operations inherit.

- insert: Updating-insert.

- update: Updating-update.

- delete: Updating-delete.

- setUserPreferences

- Personalization specific operations (cannot be instantiated by MPQF)

- getUserPreferences

- getUserActions

- getRecommendations

- Management operations (can be instantiated by MPQF)

- getRepositoryInformation 